\title{
Optimal input design for direct data-driven tuning of model-reference controllers ${ }^{\star}$
}

\author{
Simone Formentin $^{\text {a }}$, Alireza Karimi ${ }^{\text {b }}$, Sergio M. Savaresi ${ }^{\text {a }}$ \\ ${ }^{a}$ Dipartimento di Elettronica e Informazione, Politecnico di Milano, Piazza L. Da Vinci, 32, 20133 Milano (Italy) \\ e-mail: \{formentin,savaresi\}@elet.polimi.it \\ ${ }^{\mathrm{b}}$ Laboratoire d'Automatique, École Polytechnique Fédérale de Lausanne (EPFL), CH-1015 Lausanne (Switzerland) \\ e-mail: alireza.karimi@epfl.ch
}

\begin{abstract}
In recent years, direct data-driven controller tuning methods have been proposed as an alternative to the standard modelbased approach for model-reference control design. In this work, the problem of input design for noniterative direct datadriven techniques, namely Virtual Reference Feedback Tuning (VRFT) and noniterative Correlation-based Tuning (CbT), is investigated. For bounded input energy, the excitation signal is designed such that the expected value of the considered control cost is reduced. The above strategy is numerically tested on a benchmark example.
\end{abstract}

Key words: CbT, VRFT, input design, data-driven control, identification for control

\section{INTRODUCTION}

In system identification theory, optimal experiment design is about finding the operating conditions that provide the most informative data for modeling the plant. However, depending on the intended model application, the optimal experiments appear to be very different. In control applications, the model is used to design a suitable controller, and therefore the final aim for identification and input design is not to accurately describe the mathematical structure of the system, but to obtain a closed-loop behavior with some desired properties. Recently, the term "identification for control" has been introduced to refer to identification from a controloriented perspective (see [H. Hjalmarsson (2005)] for a survey). In this research area, assessing the model quality by experiment design is of primary importance, as is witnessed by a large set of contributions, see e.g. [M Gevers et al. (1986)] and [M. Gevers (1996)]. Generally, to the authors' knowledge, only the case of fullorder modeling is treated, i.e. the case where the real system belongs to the model set. The only exception is [X. Bombois et al. (2006)], where upper bounds on

\footnotetext{
* This work has been partially supported by MIUR project "New methods for Identification and Adaptive Control for Industrial Systems" and by the Austrian Center of Competence in Mechatronics.
}

modeling errors are considered. In general, parametric modeling errors influence the control design accuracy, and thus they might constitute a detrimental effect for the final control performance. As far as the authors are aware, input design for direct data-driven controller tuning, i.e. the case where a fixed-order linearly parameterized controller is directly identified from data without modeling the plant, has not been considered yet. Using these methods, the typical problems related to modeling errors can be circumvented. Moreover, these techniques can be very useful when a mathematical description of the plant is a costly and time-consuming undertaking. However, as in standard system-identification, a deep understanding of the asymptotic accuracy of the estimate is needed.

This paper attempts to obtain some insight into statistical properties of noniterative data-driven techniques, i.e. noniterative Correlation-based Tuning (CbT) and Virtual Reference Feedback Tuning (VRFT), whereof the interesting feature is that they provide a global solution to a model-reference control issue via simple least squares techniques, when the controller is linearly parameterized. The above methodologies have been only recently introduced, respectively in [A. Karimi et al. (2007)] and in [M.C. Campi et al. (2002)]. Iterative data-driven methods are instead not subjects of the present work, but it should be mentioned that an analo- 
gous study was performed for Iterative Feedback Tuning (IFT) in [R. Hildebrand et al. (2004)] and [R. Hildebrand et al. (2005)].

In this work, it will be shown that, using both the aforementioned methods, the expectation of the considered control-relevant criterion rises when measurements are noisy and that the degradation term depends on the spectral density of the input signal used for controller identification. The main goal of the paper will be then to carry out an input design methodology to reduce such a degradation effect. Throughout the theoretical analysis, only the case where the reference model is achievable will be dealt with, but the method will show effective in the numerical example also in a more realistic case.

The outline of the paper is as follows. In Section 2, preliminaries on noniterative CbT and VRFT are briefly recalled. The main analysis results and the input design procedure are discussed in Section 3, while Section 4 demonstrates the effectiveness of the above method on the benchmark simulation example proposed in [I. D. Landau et al. (1995)]. Some concluding remarks end the paper.

\section{BACKGROUNDS}

Consider the unknown LTI SISO stable plant $G\left(q^{-1}\right)$, where $q^{-1}$ denotes the backward shift operator. The objective of the model-reference control problem is to design a linear, fixed-order controller $K\left(q^{-1}, \rho\right)$, parameterized through $\rho \in \mathbb{R}^{n}$, for which the closed-loop system matches a given stable strictly proper reference model $M\left(q^{-1}\right)$. More specifically, let the controller parameterization be $K\left(q^{-1}, \rho\right)=\beta^{T}\left(q^{-1}\right) \rho$, where $\beta\left(q^{-1}\right)$ is a vector of $n$ linear discrete-time transfer operators.

Formally, the aim is to find the vector of parameters that minimizes the (filtered) $\mathcal{L}_{2}$-norm of the difference between the reference model and the achieved closed-loop system:

$$
J_{m r}(\rho)=\left\|\left(\frac{G K(\rho)}{1+G K(\rho)}-M\right) W\right\|_{2}^{2},
$$

where $W\left(q^{-1}\right)$ is a user-defined frequency-weighting filter. The goal can be interpreted as to find the minimizer of the $\mathcal{L}_{2}$-norm of the matching error signal $\varepsilon$ in Fig. 1, when the reference signal is a white noise of unit variance.

Consider now that an open-loop collection of inputoutput (I/O) data $\{u(t), y(t)\}_{t=1, \ldots, N}$ is available and let the output $y(t)$ be affected by additive noise $v(t)=H\left(q^{-1}\right) d(t)$, where $H\left(q^{-1}\right)$ is an unknown stable LTI system and $d(t)$ is a zero mean white Gaussian noise with variance of $\sigma^{2}$.

In standard "indirect" data-driven approaches, the above objective can be achieved by identifying from data a model $\hat{G}$ of the plant and designing a model-based controller $K(\hat{G})$ as

$$
K(\hat{G})=\frac{M}{\hat{G}(1-M)} .
$$

In this work, the case where the controller is directly derived from the data collection without identifying $\hat{G}$ is instead considered. In such a setting, two situations may arise, namely, the reference model can be achievable or unachievable with the given controller parameterization, according to the following definitions.

Definition 1 The reference model $M\left(q^{-1}\right)$ is said achievable with the selected controller parameterization $K\left(q^{-1}, \rho\right)=\beta^{T}\left(q^{-1}\right) \rho$ if

$$
\exists \rho_{o}: J_{m r}\left(\rho_{o}\right)=0,
$$

and it is said unachievable otherwise.

In this work, only the case of achievable reference models will be taken into account, but the strategy will be tested in both the situations in the numerical example. From now on, $K_{o}\left(q^{-1}\right)=K\left(q^{-1}, \rho_{o}\right)=\beta^{T}\left(q^{-1}\right) \rho_{o}$ will be referred to as "ideal controller" or, equivalently, "optimal controller".

\subsection{Noniterative correlation-based Tuning}

The sensitivity function of the closed-loop system with the ideal controller is given by

$$
\frac{1}{1+G K_{o}}=1-M
$$

It follows that (1) shares the same minimum of a new cost function, convex in $\rho$, defined as

$$
J(\rho)=\|(K(\rho)(1-M) G-M)(1-M) W\|_{2}^{2} .
$$

Such a minimum is also the minimum of the $\mathcal{L}_{2}$-norm of the matching error signal $\varepsilon_{c}$ in Fig. 2, when the signals are noiseless, $u(t)$ is a white noise of unit variance and used as reference signal, namely $r(t)=u(t)$. The most important observation at the basis of the $\mathrm{CbT}$ rationale is that, in the noiseless setting, the model matching error $\varepsilon_{c}(t, \rho)$ (unlike the signal $\varepsilon(t, \rho)$ defined in Fig. 1) can be directly computed from I/O data as follows:

$$
\begin{gathered}
\varepsilon_{c}(t, \rho)=W M r(t)-W(1-M) K(\rho) G r(t) \\
=W M u(t)-W(1-M)) K(\rho) y(t)
\end{gathered}
$$

and the minimizer of the $\mathcal{L}_{2}$-norm of $\varepsilon_{c}(t, \rho)$ is exactly $K_{o}\left(q^{-1}\right)$. 


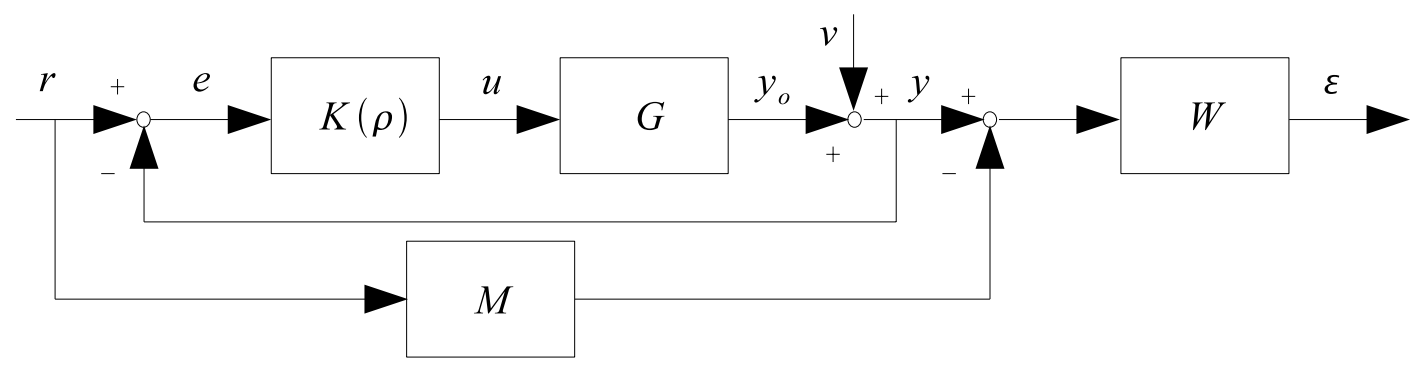

Fig. 1. Model reference control problem.

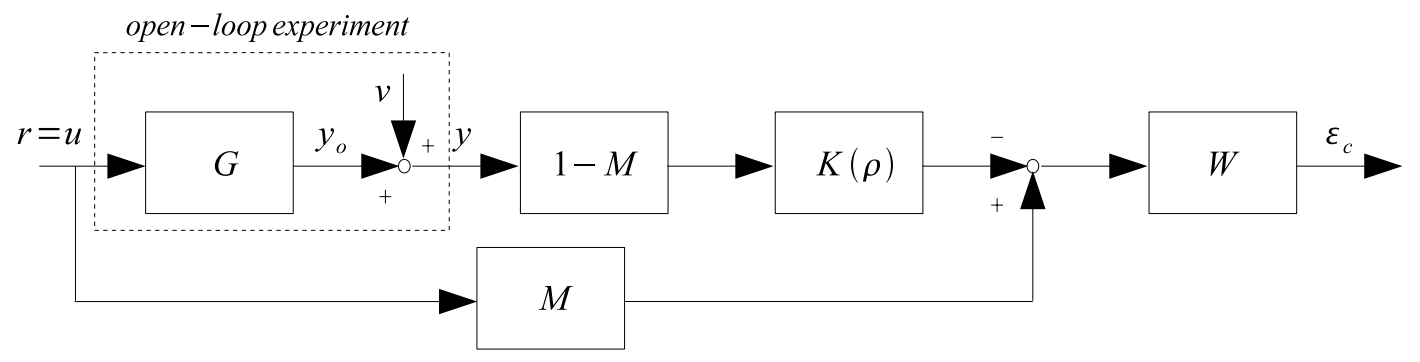

Fig. 2. Tuning scheme for Correlation-based Tuning.

When data are collected in a noisy environment, the method resorts to the correlation approach to identify the controller. Specifically, an extended instrumental variable $\zeta(t)$ correlated with $u(t)$ and uncorrelated with $v(t)$ is introduced to decorrelate the error signal $\varepsilon_{c}(t)$ and $u(t) . \zeta(t)$ is defined as

$$
\zeta(t)=[u(t+l), \ldots, u(t), \ldots, u(t-l)]^{T},
$$

where $l$ is a sufficiently large integer. The correlation function is defined as

$$
f_{N, l}(\rho)=\frac{1}{N} \sum_{t=1}^{N} \zeta(t) \varepsilon_{c}(t, \rho)
$$

and the correlation criterion as

$$
J_{N, l}(\rho)=f_{N, l}^{T}(\rho) f_{N, l}(\rho)
$$

In [K. van Heusden et al. (2011a)], it has been proven that

$$
\lim _{N, l \rightarrow \infty, l / N \rightarrow 0} J_{N, l}(\rho)=J(\rho),
$$

for any input sequence, if data in $\zeta(t)$ are prefiltered by $L_{c}\left(q^{-1}\right)$, defined as

$$
L_{c}\left(e^{-j \omega}\right)=\frac{1-M\left(e^{-j \omega}\right)}{\Phi_{u}(\omega)},
$$

where $\Phi_{u}(\omega)$ denotes the spectral density of $u(t)$. Notice that such a prefilter may be non-causal but it can be implemented off-line.

\subsection{Virtual Reference Feedback Tuning}

The idea of Virtual Reference Feedback tuning was first proposed in [G.O.Guardabassi et al. (1997)] with the name of Virtual Reference Direct Design $\left(V R D^{2}\right)$ and subsequently fixed and extended in [M.C. Campi et al. (2002)], [M.C. Campi et al. (2006)] and [S. Formentin et al. (2011)] respectively for LTI, nonlinear and LPV systems.

The main idea to minimize (1) without identifying $G\left(q^{-1}\right)$ is to build a "virtual" closed-loop system, where the input and output signals are equal to $u(t)$ and $y(t)$ and the closed-loop transfer function is assumed to correspond to $M\left(q^{-1}\right)$. From such loop, the so-called "virtual reference" $r_{v}(t)$ and "virtual error" $e_{v}(t)$ signals can be computed as $r_{v}(t)=M^{-1}\left(q^{-1}\right) y(t)$ and $e_{v}(t)=r_{v}(t)-y(t)$. The control design issue is then reduced to an identification problem, where the optimal controller is the one that generates $u(t)$ when fed by $e_{v}(t)$. The criterion to be minimized is then

$$
J_{V R}^{N}(\rho)=\frac{1}{N} \sum_{t=1}^{N}\left(u_{L}(t)-K\left(q^{-1}, \rho\right) e_{L}(t)\right)^{2},
$$

where $u_{L}(t)=L\left(q^{-1}\right) u(t), e_{L}(t)=L\left(q^{-1}\right) e_{v}(t)$ and $L\left(q^{-1}\right)$ is a suitable prefilter such that (10) is equal to the second-order Taylor expansion of (1) in the neighborhood of the minimum point (see [M.C. Campi et al. (2002)]). More specifically, the frequency response of $L\left(q^{-1}\right)$ must be such that

$$
L\left(e^{-j \omega}\right)=\frac{M\left(e^{-j \omega}\right)\left(1-M\left(e^{-j \omega}\right)\right) W\left(e^{-j \omega}\right)}{\Phi_{u}^{1 / 2}(\omega)},
$$


where $\Phi_{u}^{1 / 2}(\omega)$ denotes a spectral factor of $\Phi_{u}(\omega)$. For the final estimate not to be biased, instrumental variables are used to counteract the effect of noise (see [L. Ljung (1999)]). This method will be explained in detail in Subsection 3.2, while introducing the optimal input design strategy.

\section{OPTIMAL INPUT DESIGN}

In this section, the expectation of the considered control criteria are computed for noniterative CbT and VRFT in case of achievable reference models. Input spectrum design is then proposed to cope with the reduction of the degradation effect due to noise. Note that a signal with given spectral properties can always be generated by means of well-known spectral factorization techniques (see e.g. [A.H. Sayed et al. (2001)] and references therein).

\subsection{Noniterative correlation-based Tuning}

A preliminary asymptotic analysis of the accuracy of the controller parameter estimate for noniterative CbT has been published in [K. van Heusden et al. (2011a)]. The main result states that the expected value of the correlation criterion (7) can be approximately expressed by

$$
\begin{aligned}
& \mathbb{E}\left[J_{N, l}(\rho)\right] \approx \widetilde{J}_{N, l}(\rho)+ \\
+ & \frac{\sigma^{2}(2 l+1)}{2 \pi N} \int_{-\pi}^{\pi} \frac{|1-M|^{4}|K(\rho)|^{2}|H|^{2}|W|^{2}}{\Phi_{u}(\omega)} d \omega
\end{aligned}
$$

where $\widetilde{J}_{N, l}(\rho)$ is the noiseless counterpart of $J_{N, l}(\rho)$. For the interested reader, the formal proof of this result is briefly recalled in Appendix A. Since (8) holds and $J_{m r}(\rho)$ and $J(\rho)$ are equal at the minimum point $\rho_{0}$, Equation (12) can be rewritten (in $\rho_{o}$ ) as

$$
\begin{aligned}
& \mathbb{E}\left[J_{N, l}\left(\rho_{o}\right)\right] \approx J_{m r}\left(\rho_{o}\right)+ \\
& +\frac{\sigma^{2}(2 l+1)}{2 \pi N} \int_{-\pi}^{\pi} \frac{|1-M|^{4}\left|K_{o}\right|^{2}|H|^{2}|W|^{2}}{\Phi_{u}(\omega)} d \omega,
\end{aligned}
$$

where it is clear that the measurement noise has a detrimental effect on the control cost, because it generates a degradation term that moves the expectation of the cost function away from its ideal value. Since $l$ is fixed (and large enough) to make the first term in (12) asymptotically equal to $J_{m r}(\rho)$, then the second term in (13) might be large.

Nevertheless, Equation (13) shows that the frequency shaping of the input signal used in the experiment is strictly related to such a degradation effect (by inverse relationship) and, therefore, $\Phi_{u}(\omega)$ can be optimally selected in order to minimize it, according to the theorem presented below.

Remark Notice that the optimal input design problem can be formulated as stated above because the term $\widetilde{J}_{N, l}\left(\rho_{o}\right)$ does not asymptotically depend on the input, otherwise its effect on the approximation of $J_{m r}\left(\rho_{o}\right)$ should be also taken into account. The independence of $\widetilde{J}_{N, l}\left(\rho_{o}\right)$ from $\Phi_{u}$ is formally shown in Appendix B.

Theorem 1 Let the input energy of $u(t)$ be bounded by the application-dependent parameter $\gamma_{u}$, i.e.

$$
\int_{-\pi}^{\pi} \Phi_{u}(\omega) d \omega<\gamma_{u}
$$

The optimal expression of the input spectrum $\Phi_{u}^{o}(\omega)$ for minimization of the degradation term in (12) is given by

$$
\Phi_{u}^{o}(\omega)=\mu|1-M|^{2}\left|K_{o}\right||H||W|
$$

where

$$
\mu=\frac{\gamma_{u}}{\int_{-\pi}^{\pi}|1-M|^{2}\left|K_{o}\right||H||W| d \omega} .
$$

Proof. The proof follows the line of [L. Ljung (1999)] (Chapter 13.6) for high-order black box models. In this case, the result is not asymptotic in the controller order as the derivation of (12) does not require this assumption.

Notice that the optimal signal with spectrum $\Phi_{u}^{o}(\omega)$ cannot be directly implemented as it depends on the optimal controller $K_{o}$ and on the noise model $H\left(q^{-1}\right)$. The first problem is typical of any input design procedure (see e.g. [M. Gevers (1996)]) and, in system-identification theory, is addressed with a sequential approach, i.e. a preliminary model is first estimated from a persistently exciting set of data and such model is used to derive the optimal input. The final result is certainly suboptimal, however, it can be improved via iterative procedures (see [H. Hjalmarsson (2005)]). Analogously, also the second task is typical of any input design problem, as illustrated in [M Gevers et al. (1986)], and can be handled via preliminary knowledge or noise model identification.

\subsection{Virtual Reference Feedback Tuning}

As already mentioned in Section 2.2, instrumental variables are used in VRFT to deal with measurement noise. For implementing such a strategy, two datasets are needed as will be explained next.

For given $N$, the parameter vector are computed as

$$
\hat{\rho}_{N}=R_{N}^{-1} r_{N}
$$




$$
R_{N}=\frac{1}{N} \sum_{t=1}^{N} \phi_{2}(t) \phi_{1}^{T}(t), r_{N}=\frac{1}{N} \sum_{t=1}^{N} \phi_{2}(t) u_{L}(t) .
$$

The regressor is defined as

$$
\phi_{1}(t)=\beta\left(M^{-1}-1\right) L y_{1}(t),
$$

where $y_{1}(t)=y_{o}(t)+v_{1}(t)$ is the output of the first dataset, $y_{o}(t)=G u(t)$ is the noiseless output and $v_{1}(t)$ is the first realization of noise $v(t)$. Subsequently,

$$
\begin{aligned}
\phi_{1}(t) & =\beta\left(M^{-1}-1\right) L y_{o}(t)+\beta\left(M^{-1}-1\right) L v_{1}(t) \\
& =\phi_{o}(t)+\tilde{\phi}_{1}(t)
\end{aligned}
$$

The basic instrumental variable is defined as

$$
\phi_{2}(t)=\beta\left(M^{-1}-1\right) L y_{2}(t),
$$

where $y_{2}(t)$ is a second set of output data. Specifically, $y_{2}(t)$ might be selected as the noiseless output, i.e. $y_{2}(t)=y_{o}(t)$, that can be obtained by feeding a fullorder model of the system, if available, with the same input $u$ used in the experiment. From now on, this will be referred to as "optimal instrumental variable". Otherwise, $y_{2}(t)$ can be derived by feeding again the system with $u$; in this case, the output of this second experiment would be $y_{2}(t)=y_{o}(t)+v_{2}(t)$, where $v_{2}(t)$ is a second realization of the noise $v(t)$. In the latter case, analogously to $\phi_{1}(t)$, the instrumental variable can be rewritten as

$$
\phi_{2}(t)=\phi_{o}(t)+\tilde{\phi}_{2}(t)
$$

where

$$
\tilde{\phi}_{2}(t)=\beta\left(M^{-1}-1\right) L v_{2}(t) .
$$

Basic instrumental variables asymptotically guarantee consistent results but increase the variance of the estimate (see [T. Soderstrom et al. (2005)]). In controller identification, this fact may critically jeopardize the final performance.

As a matter of fact, consider the second order approximation of the expected value (with respect to noise) of $J_{m r}\left(\hat{\rho}_{N}\right)$ given by the Taylor expansion around the global minimum $\rho_{o}$ (recall that, in the minimum, the first order derivative is zero)

$$
\begin{gathered}
\mathbb{E}\left[J_{m r}\left(\hat{\rho}_{N}\right)\right] \approx J_{m r}\left(\rho_{o}\right)+ \\
+\frac{1}{2} \mathbb{E}\left[\left.\left(\hat{\rho}_{N}-\rho_{o}\right)^{T} \frac{\partial^{2} J_{m r}(\rho)}{\partial \rho^{2}}\right|_{\rho_{o}}\left(\hat{\rho}_{N}-\rho_{o}\right)\right] .
\end{gathered}
$$

By employing the cyclic property of the trace operator, it holds that

$$
\begin{gathered}
\mathbb{E}\left[J_{m r}\left(\hat{\rho}_{N}\right)\right] \approx J_{m r}\left(\rho_{o}\right)+ \\
+\frac{1}{2} \operatorname{tr}\left\{\mathbb{E}\left[\left(\hat{\rho}_{N}-\rho_{o}\right)\left(\hat{\rho}_{N}-\rho_{o}\right)^{T}\right] \Lambda\right\} .
\end{gathered}
$$

where $\Lambda$ is the Hessian computed in $\rho_{o}$, that has been taken out of the $\mathbb{E}[\cdot]$ argument as it is deterministic.
The previous expression can be asymptotically approximated (in distribution) by

$$
\mathbb{E}\left[J_{m r}\left(\hat{\rho}_{N}\right)\right] \approx J_{m r}\left(\rho_{o}\right)+\frac{1}{2 N} \operatorname{tr}\left\{P_{I V} \Lambda\right\}
$$

where $P_{I V}$ is the variance of the asymptotic distribution of $N \mathbb{E}\left[\left(\hat{\rho}_{N}-\rho_{o}\right)\left(\hat{\rho}_{N}-\rho_{o}\right)^{T}\right]$.

Analogously to the CbT case, the objective of reducing the effect of noise would be to reduce the degradation term in $\mathbb{E}\left[J_{m r}\left(\hat{\rho}_{N}\right)\right]$. In this paper, the input spectrum minimizing an upper bound of the above degradation term will be employed. The following theorem holds for optimal instrumental variables.

Theorem 2 Consider the case where $\phi_{2}$ is built according to (19) and $y_{2}(t)=y_{o}(t)$. Then:

- the squared effect of noise on $\mathbb{E}\left[J_{m r}\left(\hat{\rho}_{N}\right)\right]$ is upperbounded as

$$
\operatorname{tr}\left\{P_{I V} \Lambda\right\}^{2} \leq \alpha \frac{\sigma^{2} n}{2 \pi} \int_{-\pi}^{\pi} \frac{|1-M|^{8}\left|K_{o}\right|^{4}|H|^{4}|W|^{4}}{\Phi_{u}^{2}(\omega)} d \omega
$$

where

$$
\begin{gathered}
\alpha=\operatorname{tr}\left\{\int_{-\pi}^{\pi}\left|R_{o}^{-1} \Phi_{\phi_{2}}(\omega)\right|^{2} d \omega\right\}, \\
R_{o}=\lim _{N \rightarrow \infty} \frac{1}{N} \sum_{t=1}^{N} \phi_{o}(t) \phi_{o}^{T}(t)
\end{gathered}
$$

and $\Phi_{\phi_{2}}(\omega)$ is the spectral density matrix of $\phi_{2}$;

- the optimal expression of $\Phi_{u}^{o}(\omega)$ for minimization of the upper bound (25) subject to constraint (14) is given by (15), with $\mu$ as in (16).

Proof. Since the reference model is achievable, $u_{L}=$ $\phi_{o}^{T} \rho_{o}$, that is $u_{L}=\phi_{1}^{T} \rho_{o}-\tilde{\phi}_{1}^{T} \rho_{o}$. Then, $r_{N}$ in (17) becomes

$$
r_{N}=\frac{1}{N} \sum_{t=1}^{N} \phi_{2}(t) \phi_{1}^{T}(t) \rho_{o}-\frac{1}{N} \sum_{t=1}^{N} \phi_{2}(t) \tilde{\phi}_{1}^{T}(t) \rho_{o}
$$

and $\sqrt{N}\left(\hat{\rho}_{N}-\rho_{o}\right)$ can be rewritten as

$$
\sqrt{N}\left(\hat{\rho}_{N}-\rho_{o}\right)=R_{N}^{-1} \frac{1}{\sqrt{N}} \sum_{t=1}^{N} \phi_{2}(t) \tilde{\phi}_{1}^{T}(t) \rho_{o} .
$$

According to [L. Ljung (1999)], as $N \rightarrow \infty$,

$$
\frac{1}{\sqrt{N}} \sum_{t=1}^{N} \phi_{2}(t) \tilde{\phi}_{1}^{T}(t) \rho_{o} \rightarrow \mathcal{N}\left(0, P_{o}\right)
$$




$$
P_{o}=\lim _{N \rightarrow \infty} \frac{1}{N} \mathbb{E}\left[\sum_{t=1}^{N} \sum_{s=1}^{N} \phi_{2}(t) \tilde{\phi}_{1}^{T}(t) \rho_{o} \rho_{o}^{T} \tilde{\phi}_{1}(s) \phi_{2}^{T}(s)\right] .
$$

Following the rationale in [T. Soderstrom et al. (1983)] (Appendix A8.1, pages 285-286), since $n=\operatorname{dim}(\rho)=$ $\operatorname{dim}\left(\phi_{2}\right)$, the variance expression can be rewritten as

$$
\begin{gathered}
P_{o}=\sigma^{2} \mathbb{E}\left[F\left(q^{-1}\right) \phi_{2}(t)\right]\left[F\left(q^{-1}\right) \phi_{2}(t)\right]^{T}, \\
F=K_{o}(1-M)^{2} H W \Phi_{u}^{-1 / 2} .
\end{gathered}
$$

Subsequently, $P_{I V}=R_{o}^{-1} P_{o} R_{o}^{-1}$, where $R_{o}$ is as in (27), because $R_{N} \rightarrow R_{o}$ for $N \rightarrow \infty$. Since the optimal filter (11) is designed so as to make the noiseless version of $J_{v r}$ equal to the second order approximation of $J_{m r}$ in the minimum point (see [M.C. Campi et al. (2002)]), it holds that

$$
\Lambda=\left.\frac{\partial^{2} J_{m r}(\rho)}{\partial \rho^{2}}\right|_{\rho_{o}}=R_{o}
$$

and, as a consequence,

$$
\operatorname{tr}\left\{P_{I V} \Lambda\right\}=\operatorname{tr}\left\{R_{o}^{-1} P_{o}\right\} .
$$

The Parseval counterpart of (34) is

$$
\operatorname{tr}\left\{\frac{\sigma^{2}}{2 \pi} \int_{-\pi}^{\pi}|F|^{2} R_{o}^{-1} \Phi_{\phi_{2}}(\omega) d \omega\right\} .
$$

Now recall the Cauchy-Schwarz inequality for matrixvalued functions in [A. Bultheel (1982)], for which, given two matrices $A$ and $B$ and a nonnegative functional < $\cdot, \cdot>$, it holds that

$$
\operatorname{tr}\{<A, B>\}^{2} \leq \operatorname{tr}\{<A, A>\} \operatorname{tr}\{<B, B>\} .
$$

If the above result is applied to $(35)$ where $<\cdot, \cdot>$ is selected as the integral operator, $A=\sigma^{2}|F|^{2} I_{n}$ (where $I_{n}$ is the identity matrix of dimension $n$ ) and $B=R_{o}^{-1} \Phi_{\phi_{2}}$, then the squared Parseval counterpart of (34) is upperbounded as

$$
\begin{gathered}
\operatorname{tr}\left\{R_{o}^{-1} P_{o}\right\}^{2} \leq \frac{\sigma^{2} n}{2 \pi} \int_{-\pi}^{\pi}|F|^{4} d \omega \operatorname{tr}\left\{\int_{-\pi}^{\pi} \Gamma(\omega) d \omega\right\}, \\
\Gamma(\omega)=\left|R_{o}^{-1} \Phi_{\phi_{2}}(\omega)\right|^{2} .
\end{gathered}
$$

By recalling the expression of $F$ in (32) and defining

$$
\alpha=\operatorname{tr}\left\{\int_{-\pi}^{\pi} \Gamma(\omega) d \omega\right\},
$$

the first statement of the theorem is finally proven. Concerning the second statement, it should be first noticed that $R_{o}$ asymptotically does not depend on $\Phi_{u}$ for the same reason of $\tilde{J}_{N, l}$ in CbT, i.e., in few words, because $\phi_{o}$ is prefiltered by $L$ (that depends on the inverse of $\Phi_{u}^{1 / 2}$ ); further, since by hypotheses $\phi_{2}=\phi_{o}$,

$$
\Phi_{\phi_{2}}=\beta \beta^{T}|1-M|^{4}|W|^{2}|G|^{2}
$$

and $\Phi_{\phi_{2}}$ does not depend on $\Phi_{u}$ either. Therefore, analogously to Theorem 1, the proof of the second statement simply follows the line of [L. Ljung (1999)] (Chapter 13.6) to find the analytical minimum of the integral term in (25).

\section{Remarks}

- A very interesting observation is that the optimal spectra for CbT and VRFT in the proposed formulation are exactly the same.

- Also in identification for control, quantitative measurements of the expected value of the control criterion can be derived from variance of $G$ and $K$ (see e.g. [H. Hjalmarsson (2005)], [M. Gevers (1996)] and [M Gevers et al. (1986)]) but computations become complicated.

- It should be noticed that, when the instrumental variable of VRFT is selected using a second experiment, the result in Theorem 2 no longer holds. In Section 4, the effectiveness of this input choice with instrumental variable built from a second experiment will be numerically shown.

- As already mentioned in Introduction, a similar analysis has been done for Iterative Feedback Tuning (IFT) in [R. Hildebrand et al. (2004)] and [R. Hildebrand et al. (2005)]. In such a study, the standard control scheme for disturbance rejection is taken into account and the expression of a degradation term - due to measurement noise - on the expected value of the criterion

$$
J_{I F T}(\rho)=\frac{1}{2} \mathbb{E}\left[y(t, \rho)^{2}+\lambda u(t, \rho)^{2}\right]
$$

can be derived. More specifically, under the assumptions that $\lambda=0$, the approximation (3) holds and the controller is linearly parameterized as in Section 2 , then, for the $m$-th iteration,

$$
\begin{gathered}
\mathbb{E}\left[J_{I F T}\left(\hat{\rho}_{m}\right)\right]=J_{I F T}\left(\rho_{o}\right)+\frac{a^{2} \sigma^{2}}{(2 a-1) 4 \pi} \times \\
\times \int_{-\pi}^{\pi} \frac{|1-M|^{4}|H|^{2}}{\Phi_{r}} \operatorname{tr}\left\{R_{I F T}^{-1}\left(\rho_{o}\right) \beta \beta^{T}\right\} d \omega,
\end{gathered}
$$

where $R_{I F T}\left(\rho_{o}\right)$ denotes the Hessian of $J_{I F T}$ around $\rho_{o}, \Phi_{r}$ is the spectrum of the reference signal to be selected (the "input signal") and $a$ is a positive constant determining the step size $\gamma_{m}=a / m$. 
The aim of experiment design in this framework is again to minimize the degradation term due to noise. Notice that, like in noniterative $\mathrm{CbT}$, the additional term directly depends on $|1-M|^{4}|H|^{2}$ and on the inverse of the input filtering inside the integral. However, such a result is different from the ones presented herein because the term $\left|K_{o}\right|^{2}$ is missing inside the integral (the signal routing is slightly different in the noise rejection scheme) and only the trace of its parameterization $\beta$ occurs. Concerning the frequency weighting, $W$ is missing in the original formulation but can be easily added to $J_{I F T}$ to make it appear inside the integral term.

\section{A SIMULATION EXAMPLE: THE FLEXI- BLE TRANSMISSION SYSTEM}

The example proposed herein for testing the above input design strategy is the flexible transmission system introduced as a benchmark for digital control design in [I. D. Landau et al. (1995)]. Firstly, the case of achievable reference model will be considered, so as to apply the theoretical results presented herein. In the VRFT case, both optimal instrumental variable and instrumental variable built from two experiments will be employed, to show that the proposed input is suitable in both the cases. Secondly, it will be (numerically) shown that the control-oriented input signals might be better than standard PRBS also when the desired closed-loop behavior is unachievable.

The plant is described by the discrete-time model

$$
\begin{gathered}
G\left(q^{-1}\right)=\frac{0.28261 q^{-3}+0.50666 q^{-4}}{A\left(q^{-1}\right)}, \\
A\left(q^{-1}\right)=1-1.41833 q^{-1}+1.58939 q^{-2}+ \\
-1.31608 q^{-3}+0.88642 q^{-4},
\end{gathered}
$$

where the sampling time is $T_{s}=0.05 \mathrm{~s}$. The measurement noise is supposed to be white and such that the signal-to-noise ratio is 5 , that is $H\left(q^{-1}\right)=\operatorname{var}\left[y_{o}(t)\right] / 5$, where $\operatorname{var}\left[y_{o}(t)\right]$ is the variance of $y_{o}(t)$. Moreover, the frequency-weighting function $W\left(q^{-1}\right)=1$, the number of samples is $N=1000$ and $\gamma_{u}=1$. Finally, the set of available controllers is

$$
K(\rho)=\frac{\rho_{0}+\rho_{1} q^{-1}+\rho_{2} q^{-2}+\rho_{3} q^{-3}+\rho_{4} q^{-4}+\rho_{5} q^{-5}}{1-q^{-1}} .
$$

\subsection{Achievable reference model}

Define the control objective as a reference model that allows the perfect matching to be achieved, i.e.

$$
M\left(q^{-1}\right)=\frac{G\left(q^{-1}\right) K\left(q^{-1}, \rho_{o}\right)}{1+G\left(q^{-1}\right) K\left(q^{-1}, \rho_{o}\right)},
$$

where the optimal controller is in the controller set and its parameters are

$$
\rho_{o}=[0.2045,-0.2715,0.2931,-0.2396,0.1643,0.0084] \text {. }
$$

The following steps will be followed for all the datadriven design procedures:

- a first experiment with PRBS input excitation is performed on the system;

- a preliminary estimate of the controller is carried out via standard CbT/VRFT; a FIR model of the system of order 200 is also derived for the design using optimal instrumental variables;

- the optimal input spectrum is computed according to Section 3, using the preliminary estimate of the controller found at the previous step;

- a second experiment with optimal input excitation is performed on the system;

- the optimal controller is given by CbT/VRFT design using the optimal experiment data.

With simulation parameters above, the optimal input spectrum for CbT and VRFT is shaped as illustrated in Fig. 3, where the magnitude plot of $G$ is also showed. Notice that the input energy is low in the frequencies corresponding to the resonances, whereas it is higher around the desired bandwidth and at high frequencies, where the desired sensitivity function $1-M\left(q^{-1}\right)$ is high.

To verify the effectiveness of the proposed strategy, a

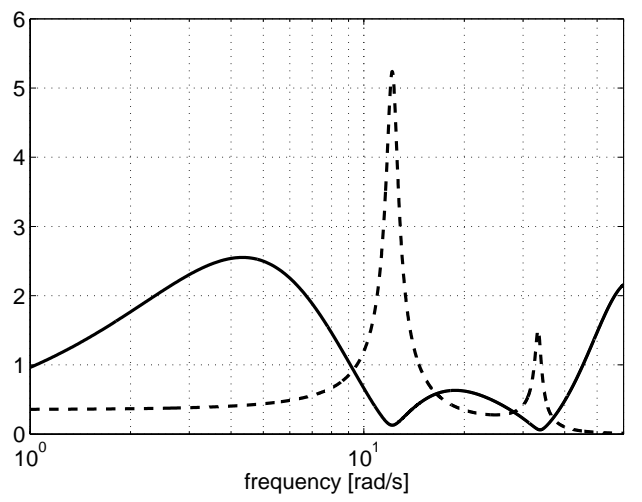

Fig. 3. Normalized magnitude plot of $G$ (dashed) and optimal input spectrum for CbT and VRFT (solid) on the linear scale.

Monte-Carlo simulation with 100 running experiments 
is performed, using a different noise realization for each experiment, and an estimate of $\mathbb{E}\left[J_{m r}\left(\hat{\rho}_{N}\right)\right]$ in the minimum $\hat{\rho}_{N}$ is computed by sample mean, to compare PRBS and optimal input. Final results are illustrated in Table 1, whereas Fig. 4 and 5 show the accuracy of the magnitude plots. In each figure, the ideal closed-loop behavior $M\left(q^{-1}\right)$ is also shown. For CbT, the length of the instrumental variable vector is $l=35$, which corresponds to the length of the impulse response of $M$. Notice that, when VRFT requires two sets of data for building the instrumental variable, each experiment is made of $N / 2=500$ samples for a fair comparison between the methods.

From numerical results, it is clear that the use of the

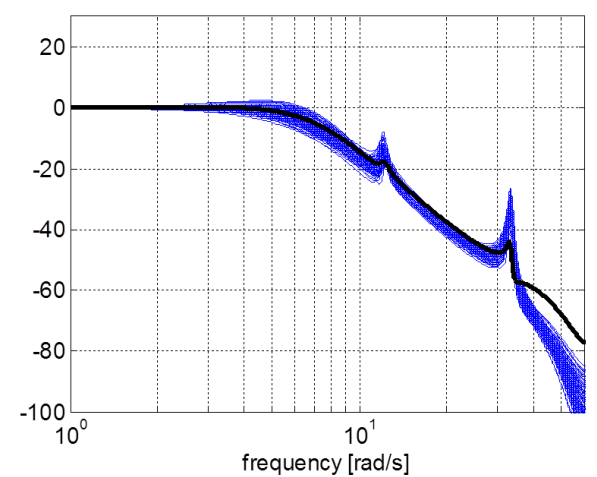

(a)

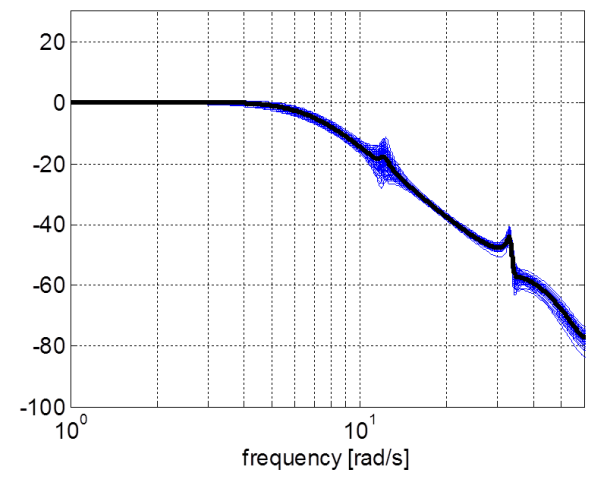

(b)

Fig. 4. Magnitude Bode plots of $M$ (black line), achieved closed-loop performance with PRBS input for the 100/100 stabilizing CbT controllers (blue lines, above), with optimal input signal for 100/100 stabilizing controllers (blue lines, below).

optimal input not only improves the closed-loop performance but also makes CbT and VRFT (with any kind of instrumental variable) comparable in terms of statistical behavior. Moreover, the degrade of performance due to the use of a double experiment is small. Obviously, such advantages are not completely costless as a first experimental session is required to estimate $K_{o}$ and $H$ required by (15). However, the proposed approach seems

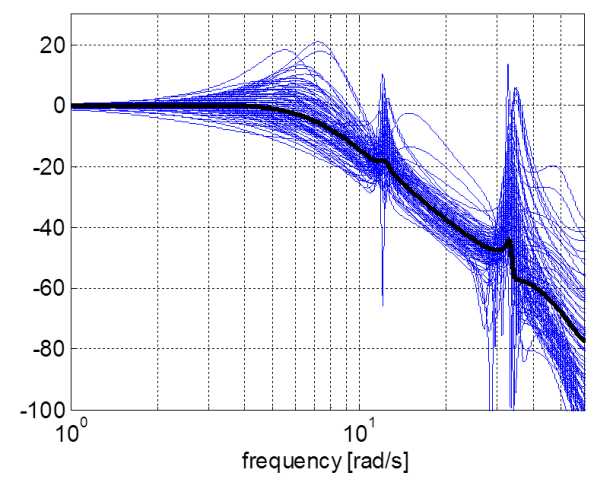

(a)

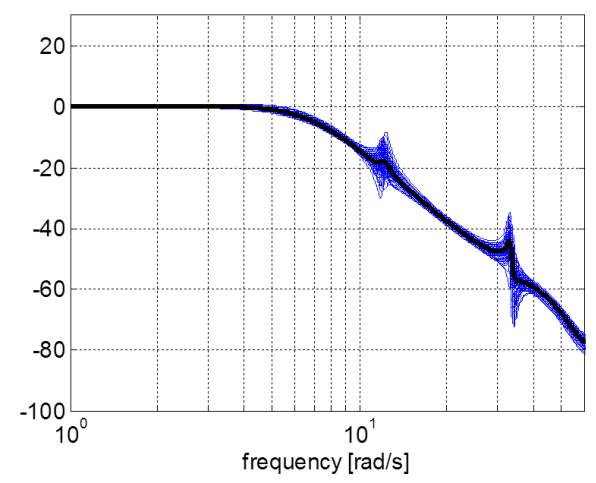

(b)

Fig. 5. Magnitude Bode plots of $M$ (black line), achieved closed-loop performance with PRBS input for the 95/100 stabilizing VRFT controllers (blue lines, above), with optimal input signal for 100/100 stabilizing controllers (blue lines, below). Two experiments are used to build the instrumental variable.

Table 1

Mean values (100 iterations) of the achieved performance $J_{m r}$ if the reference model (42) is selected. For VRFT design, results with both optimal instrumental variables ("opt IV") and instruments built from two datasets ("2 exp") are shown.

\begin{tabular}{c||c|c}
\hline & PRBS & optimal input \\
\hline \hline CbT & 0.0527 & 0.0273 \\
\hline VRFT (opt IV) & 0.0477 & 0.0154 \\
\hline VRFT (2 exp) & 0.1769 & 0.0290 \\
\hline
\end{tabular}

to the authors a very good trade-off for all applications where experiments are not too costly and high accuracy is required.

Finally notice that, due to high variance of the parameters, some VRFT controllers may destabilize the system, e.g. in the example of Fig. 5, 5/100 controllers are destabilizing. Reduction of the degradation term via optimal input design also allows one to reduce the probability of obtaining such controllers without adding any additional (and conservative) stability constraint (see e.g. [K. van Heusden et al. (2011a)]) to the design pro- 
Table 2

Mean values (100 iterations) of the achieved performance $J_{m r}$ if the reference model (43) is selected. For VRFT design, results with both optimal instrumental variables ("opt IV") and instruments built from two datasets ("2 exp") are shown.

\begin{tabular}{c||c|c}
\hline & PRBS & optimal input \\
\hline \hline CbT & 0.1277 & 0.0800 \\
\hline VRFT (opt IV) & 0.0861 & 0.0775 \\
\hline VRFT (2 exp) & 0.1899 & 0.0814 \\
\hline
\end{tabular}

cedure.

\subsection{Unachievable reference model}

The same tests of the previous subsection can be carried out on the system (40), in the case where the controller structure is not flexible enough to achieve a given reference model, e.g. when the controller is again as in Subsection 4.1 but the reference model is instead selected as

$$
M\left(q^{-1}\right)=\frac{(1-\alpha)^{2} q^{-3}}{\left(1-\alpha q^{-1}\right)^{2}}, \alpha=e^{-10 T_{s}} .
$$

As already illustrated in [M.C. Campi et al. (2002)], there is no controller in the given set that yields $J_{m r}(\rho)=0$. Recalling that, being $M$ different from the previous case, also the control-relevant input spectrum will be accordingly modified, the input sequence can still be designed according to the procedure illustrated in this work. Specifically, the same procedure illustrated in the previous subsection is employed.

In Table 2, final results are shown, according to the same rationale of Table 1, whereas Fig. 6 and 7 illustrate the magnitude plots of the Monte-Carlo runs. Numerical results point out how the use of a suited input signal might outperform the standard PRBS solution also in this situation.

\section{CONCLUSIONS AND FUTURE WORKS}

In this work, statistical properties of direct data-driven controller tuning have been analyzed and optimal input design have been proposed to increase closed-loop performance. Specifically, the innovative contributions of the paper can be summarized as follows:

- CbT and VRFT have been demonstrated to have the same problem for large $N$; specifically, the expected value of the considered control-relevant cost function contains a degradation term when data are noisy;

- the final cost functions and the input spectrum expressions have been shown to be connected in a straightforward way. The spectrum can then be directly exploited to improve the closed-loop performance;

- an optimal experiment design problem has been formulated for each technique and the analytical expression of the optimal input signal has been provided; an

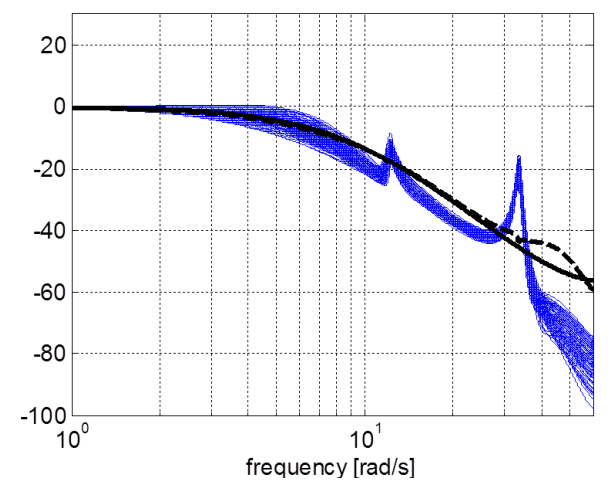

(a)

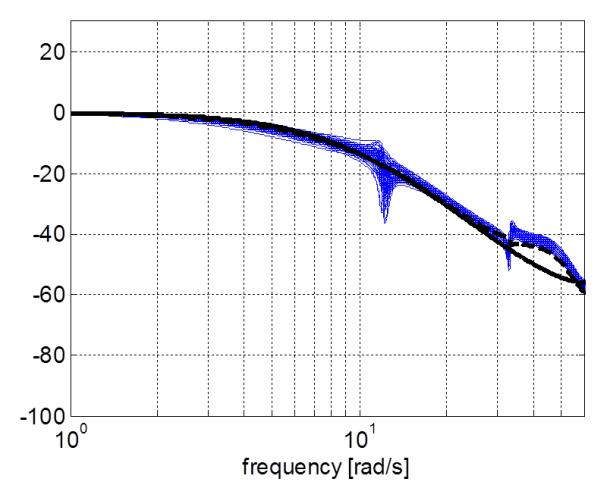

(b)

Fig. 6. Magnitude Bode plots of unachievable $M$ (black solid line), noiseless estimate (black dashed line), achieved closed-loop performance with PRBS input for the 100/100 stabilizing CbT controllers (blue lines, above), with optimal input signal for 100/100 stabilizing controllers (blue lines, below).

open-loop experiment with such an input allows the degradation effect to be reduced;

- the benchmark numerical example has shown to behave much better when the control-relevant input is used, also in case of unachievable reference model; moreover, destabilizing controllers are no longer obtained.

Future work will focus on the theoretical analysis of the performance in case of unachievable reference model.

\section{References}

[M.C. Campi et al. (2002)] M.C. Campi, A. Lecchini and S.M. Savaresi, Virtual Reference Feedback Tuning: a direct method for the design of feedback controllers, Automatica, 38(8), 2002.

[A. Sala et al. (2005)] A. Sala and A. Esparza, Extensions to virtual reference feedback tuning: A direct method for the design of feedback controllers, Automatica, 41, 2005.

[A. Karimi et al. (2007)] A. Karimi, K. van Heusden, and D. Bonvin, Noniterative Data-driven Controller Tuning Using 


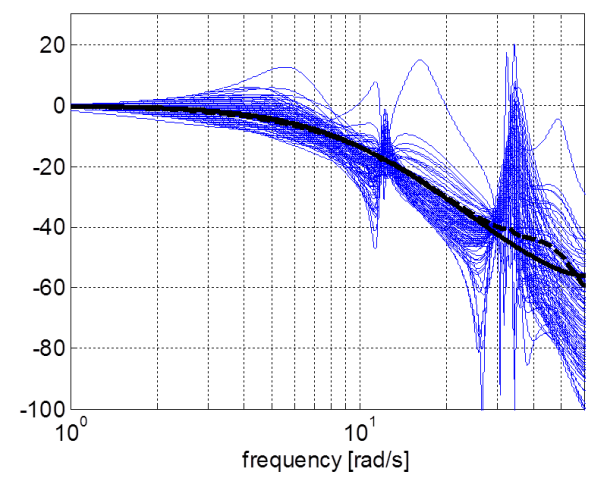

(a)

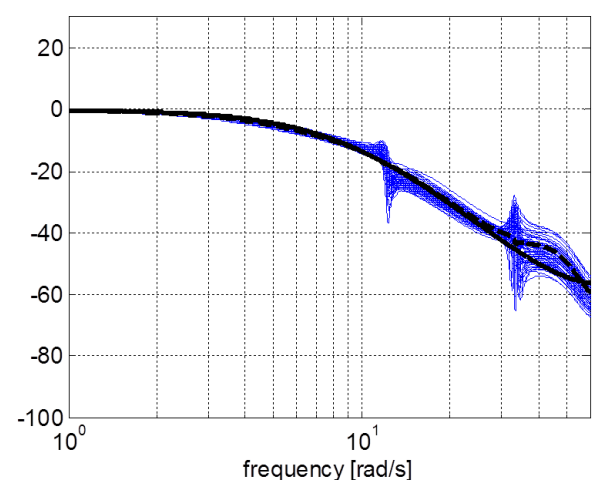

(b)

Fig. 7. Magnitude Bode plots of unachievable $M$ (black line), noiseless estimate (black dashed line), achieved closed-loop performance with PRBS input for the 93/100 stabilizing VRFT controllers (blue lines, above), with optimal input signal for 100/100 stabilizing controllers (blue lines, below). Two experiments are used to build the instrumental variable.

the Correlation Approach, European Control Conference, $200 \%$.

[K. van Heusden et al. (2011a)] K. van Heusden, A. Karimi and D. Bonvin, Data-driven model reference control with asymptotically guaranteed stability, Int J. Adapt. Control Signal Process., 2011.

[K. van Heusden et al. (2011b)] K. van Heusden, A. Karimi and T. Soderstrom, On identification methods for direct datadriven controller tuning, Int J. Adapt. Control Signal Process., 2011.

[I. D. Landau et al. (1995)] I. D. Landau, D. Rey, A. Karimi, A. Voda, and A. Franco, A flexible transmission system as a benchmark for robust digital control, European Journal of Control, 1995.

[S. Formentin et al. (2011)] S. Formentin and S.M. Savaresi, Virtual Reference Feedback Tuning for linear parametervarying systems, IFAC World Congress, Milan, 2011.

[M.C. Campi et al. (2006)] M.C. Campi and S.M. Savaresi, Direct Nonlinear Control Design: The Virtual Reference Feedback Tuning (VRFT) Approach, IEEE Transactions on Automatic Control, Vol. 51, No. 1, January 2006.

[G.O.Guardabassi et al. (1997)] G.O. Guardabassi and S.M. Savaresi, Approximate feedback linearization of discretetime non-linear systems using Virtual Input Direct Design,
Systems 83 Control Letters, vol.32, pp.63-74, 1997.

[H. Hjalmarsson (2002)] H. Hjalmarsson, Iterative feedback tuning - an overview, Int J. Adapt. Control Signal Process., 16, 2002.

[A. Karimi et al. (2004)] A. Karimi, L. Miskovic, and D. Bonvin, Iterative correlation-based controller tuning, Int J. Adapt. Control Signal Process., 18(8), 2004.

[M. Gevers (1996)] M. Gevers and L. Ljung, Identification for control, Annual Reviews in Control 20: 95-106, 1996.

[L. Ljung (1999)] L. Ljung, System identification: theory for the user, Prentice-Hall, Englewood Cliffs, NJ, 1999.

[M Gevers et al. (1986)] M. Gevers and L. Ljung, Optimal experiment designs with respect to the intended model application, Automatica, 22, 543-554, 1986.

[H. Hjalmarsson (2005)] H. Hjalmarsson, From experiment design to closed-loop control, Automatica 41 393438, 2005.

[T. Soderstrom et al. (1983)] T. Soderstrom and P.G. Stoica, Instrumental variable methods for system identification, Springer-Verlag, New York, 1983.

[T. Soderstrom et al. (2005)] T. Soderstrom and M. Hong, Identification of dynamic errors-in-variables systems with periodic data, 16th IFAC World Congress, Prague, Czech Republic, 2005.

[A.H. Sayed et al. (2001)] A.H. Sayed and T. Kailath, A survey of spectral factorization methods, Numerical Linear Algebra with Applications 8: 467-496.

[X. Bombois et al. (2006)] X. Bombois and M. Gilson, Cheapest identification experiment with guaranteed accuracy in the presence of undermodeling, 14th IFAC Symposium on System Identification, pp. 505-510, Newcastle, March 2006.

[R. Hildebrand et al. (2004)] R. Hildebrand, A. Lecchini, G. Solari, and M. Gevers, Prefiltering in iterative feedback tuning: optimization of the prefilter for accuracy, IEEE Transactions on Automatic Control, 49(10):18011806, 2004.

[R. Hildebrand et al. (2005)] R. Hildebrand, A. Lecchini, G. Solari, and M. Gevers, Optimal prefiltering in iterative feedback tuning, IEEE Transactions on Automatic Control, 50(8):1196 1200, 2005.

[A. Bultheel (1982)] A. Bultheel, Inequalities in Hilbert modules of matrix-valued functions, Proceedings of the American mathematical society, 85(3):369 372, 1982.

\section{Appendix}

\section{A. Proof of (12)}

Consider again the scheme in Fig. 2. In the noisy case, the expression of $\varepsilon_{c}$ becomes

$$
\begin{aligned}
\varepsilon_{c}(t, \rho)= & W[M-(1-M) K(\rho) G] u(t)+ \\
& -W K(\rho)(1-M) H d(t),
\end{aligned}
$$

that is

$$
\varepsilon_{c}(t, \rho)=D(\rho) u(t)-C(\rho) d(t),
$$

with obvious definitions for filters $D(\rho)$ and $C(\rho)$.

Now notice that $d_{C}(t, \rho)=C(\rho) d(t)$ can be written as

$$
d_{C}(t, \rho)=\sum_{k=0}^{\infty} c_{k}(\rho) d(t-k)
$$


where $c_{k}(\rho)$ is the impulse response of $C(\rho)$. The vector of random variables

$$
X_{N}(\rho)=\frac{1}{\sqrt{N}} \sum_{t=1}^{N} \zeta(t) d_{C}(t, \rho)
$$

converges in distribution to a normal distribution with zero mean and variance

$$
\begin{gathered}
P_{X}(\rho)=\lim _{N \rightarrow \infty} \mathbb{E}\left[X_{N}(\rho) X_{N}^{T}(\rho)\right]= \\
=\sigma^{2} \lim _{N \rightarrow \infty} \frac{1}{N} \sum_{t=1}^{N} \mathbb{E}\left[\tilde{\zeta}(t) \tilde{\zeta}^{T}(t)\right], \\
\tilde{\zeta}(t)=C(\rho) L_{c}[u(t+l), \ldots, u(t), \ldots, u(t-l)]^{T} .
\end{gathered}
$$

Now, the expectation of the correlation cost criterion is

$$
\mathbb{E}\left[J_{N, l}(\rho)\right]=\mathbb{E}\left[\frac{1}{N^{2}} \sum_{t=1}^{N} \zeta^{T}(t) \varepsilon_{c}(t, \rho) \sum_{t=1}^{N} \zeta(t) \varepsilon_{c}(t, \rho)\right],
$$

that is, following the expression of $\varepsilon_{c}$,

$$
\begin{gathered}
\mathbb{E}\left[J_{N, l}(\rho)\right]=\mathbb{E}\left[\frac{1}{N^{2}} \sum_{t=1}^{N} \zeta^{T}(t) D(\rho) u(t) \sum_{t=1}^{N} \zeta(t) D(\rho) u(t)\right]+ \\
-2 \mathbb{E}\left[\frac{1}{N^{2}} \sum_{t=1}^{N} \zeta^{T}(t) D(\rho) u(t) \sum_{t=1}^{N} \zeta(t) C(\rho) d(t)\right]+ \\
+\mathbb{E}\left[\frac{1}{N^{2}} \sum_{t=1}^{N} \zeta^{T}(t) C(\rho) d(t) \sum_{t=1}^{N} \zeta(t) C(\rho) d(t)\right]= \\
=\tilde{J}_{N, l}(\rho)-0+\frac{1}{N} \sum_{t=1}^{N} \mathbb{E}\left[X_{N}^{T}(\rho) X_{N}(\rho)\right] .
\end{gathered}
$$

For large $N$, the variance of $X_{N}$ tends to $P_{X}$, therefore, according to the cyclic property of the trace operator, it is possible to approximate $\mathbb{E}\left[J_{N, l}(\rho)\right]$ as

$$
\mathbb{E}\left[J_{N, l}(\rho)\right] \approx \tilde{J}_{N, l}(\rho)+\operatorname{tr}\left\{P_{X}(\rho)\right\} .
$$

To compute the last term, notice that the diagonal elements of $P_{X}$ are equal to $\sigma^{2} R_{u_{C L_{c}}}(0)$, where $R_{u_{C L_{c}}}(\tau)$ is the autocorrelation function of $C(\rho) L_{c} u(t)$. Then,

$$
\mathbb{E}\left[J_{N, l}(\rho)\right] \approx \tilde{J}_{N, l}(\rho)+\frac{2 l+1}{N} \sigma^{2} R_{u_{C L_{c}}}(0)
$$

and, using the Parseval theorem,

$\mathbb{E}\left[J_{N, l}(\rho)\right] \approx \tilde{J}_{N, l}(\rho)+\frac{\sigma^{2}(2 l+1)}{2 \pi N} \int_{-\pi}^{\pi}\left|C(\rho) L_{c}\right|^{2} \Phi_{u}(\omega) d \omega$.

Expression (12) is derived from (45) by simply replacing $C(\rho)$ and $L_{C}$ with $W K(\rho)(1-M) H$ and (9), respectively.
B. Proof that $\widetilde{J}_{N, l}(\rho)$ in (12) is asymptotically independent from $\Phi_{u}$

From the proof of (12), we have

$$
\widetilde{J}_{N, l}(\rho)=\left[\frac{1}{N^{2}} \sum_{t=1}^{N} \zeta^{T}(t) u_{D}(t, \rho) \sum_{t=1}^{N} \zeta(t) u_{D}(t, \rho)\right]
$$

Note that, since no term in the above equation depends on noise, the expectation is removed. Let the crosscorrelation function between $u(t)$ and $\varepsilon(t)=L_{c} D(\rho) u(t)$ be defined as:

$$
R_{u \varepsilon}(\tau)=\lim _{N \rightarrow \infty} \frac{1}{N} \sum_{t=1}^{N} u(t-\tau) \varepsilon(t)
$$

Then for large $N$ we get:

$$
\frac{1}{N} \sum_{t=1}^{N} \zeta^{T}(t) u_{D}(t, \rho) \approx\left[R_{u \varepsilon}(-l), \cdots, R_{u \varepsilon}(l)\right]
$$

It follows that

$$
\widetilde{J}_{N, l}(\rho) \approx \sum_{\tau=-l}^{l} R_{u \varepsilon}^{2}(\tau)
$$

Since $\varepsilon$ is obtained by filtering of $u$ with a finite order stable filter, it is reasonable to assume that $R_{u \varepsilon}(\tau)=0$ for $\tau>\tau_{m}$. Thus, for $l>\tau_{m}$, using Parseval's theorem, we have:

$$
\sum_{\tau=-l}^{l} R_{u \varepsilon}^{2}(\tau)=\frac{1}{2 \pi} \int_{-\pi}^{\pi}\left|L_{c}\right|^{2}|D(\rho)|^{2} \Phi_{u}^{2}(\omega) d \omega .
$$

Replacing $L_{c}$ with (9), it follows that

$$
\widetilde{J}_{N, l}(\rho) \approx \frac{1}{2 \pi} \int_{-\pi}^{\pi}|1-M|^{2}|D(\rho)|^{2} d \omega,
$$

that is, $\widetilde{J}_{N, l}(\rho)$ does not (asymptotically) depend on $\Phi_{u}(\omega)$. Notice that this is due to the frequency shaping of the filter $L_{c}$. 